\title{
Febre Infantil e seu Manejo pelos Pais: Análise Quantitativa
} Childhood Fever and its Management by Parents: A Quantitative Analysis

\section{RESUMO}

Objetivo: verificar o conhecimento, crenças, fontes de informação, práticas e atitudes dos pais/cuidadores no manejo da febre infantil. Material e Métodos: estudo descritivo transversal, de caráter quantitativo. A amostra composta por 286 pais/cuidadores que procuraram o serviço de urgência de um Hospital Infantil em Minas Gerais com relato de febre na criança. Resultados: $45,6 \%$ dos pais possuiam o ensino médio, $54 \%$ ganhavam até um salário mínimo e $42 \%$ tinham um filho. $70 \%$ possuiam termômetro, $48,4 \%$ não observaram o tempo adequado para retirada do termômetro de mercúrio e $29,4 \%$ verificavam pela palpação. $34,3 \%$ conceituaram febre a partir de $37,5^{\circ} \mathrm{C} .96,3 \%$ administravam antitérmicos, $50,5 \%$ dipirona e $39,3 \%$ paracetamol. $83,3 \%$ desconheciam os efeitos adversos. $14 \%$ administravam antibióticos. $70 \%$ consideravam que a febre trazia malefícios e que o pior dano é a convulsão. Para detectar a febre os entrevistados utilizaram o termômetro e a palpação. Consideram a febre perigosa por acreditarem provocar convulsão, sonolência, prostração, desidratação e danos cerebrais. Para controle da febre utilizavam métodos farmacológicos e não farmacológicos. Como fontes de informação, acessam a internet, livros, televisão, jornais, profissionais de saúde, amigos e parentes. Conclusão: Os participantes possuíam perfil socioeconômico, medos, crenças e práticas de manejo da febre similares. Em algumas situações demonstraram conhecimento e percepções limitadas ocasionando em atitudes errôneas frente à febre. As concepções direcionam as condutas e práticas no manejo da febre. A febre fobia persiste, pais/ cuidadores demonstram insegurança para cuidar da criança febril.

DESCRITORES

Febre.Conhecimento.Criança.Percepção.Atitude.

\begin{abstract}
Objective: To verify the knowledge, beliefs, sources of information, practices and attitudes of the parents/caregivers in the management of childhood fever. Material and Methods: This was a descriptive, cross-sectional study, with a quantitative approach. The sample was composed of 286 parents/caregivers who sought the emergency service of a Children's Hospital in Minas Gerais with complaints of fever in their children. Results: $45.6 \%$ of parents had high school education level; $54 \%$ earned up to one minimum wage; and $42 \%$ had one child. A total of $70 \%$ of respondents had a thermometer, $48.4 \%$ did not observe the adequate time to remove the mercury thermometer; and $29.4 \%$ of them verified fever by palpation. $34.3 \%$ reported fever at $37.5{ }^{\circ} \mathrm{C} ; 96.3 \%$ administered antipyretics; $50.5 \%$ administered dipyrone and $39.3 \%$ paracetamol; $83.3 \%$ were unaware of the adverse effects; $14 \%$ administered antibiotics; $70 \%$ considered that the fever was harmful and that the worst damage is the seizure. To detect fever, the interviewees used the thermometer and palpation They consider the fever dangerous because they believe it may cause convulsion, drowsiness, prostration, dehydration and brain damage. For fever control, they used pharmacological and nonpharmacological methods. As sources of information, they access the internet, books, television, newspapers, health professionals, friends and relatives. Conclusion: The participants had similar socioeconomic profiles, fears, beliefs and practices of fever management. They showed limited knowledge and perceptions leading to erroneous attitudes towards fever management. Conceptions guide conduct and practices in the management of fever. The phobia of fever persists, and parents/caregivers show insecurity to care for the febrile child.
\end{abstract}

\section{DESCRIPTORS}

Fever.Knowledge.Child.Perception.Attitude.

1 Docente Mestre do Programa de Residência Multiprofissional em urgência e trauma do Hospital Municipal José Lucas Filho. Contagem. Minas Gerais. Brasil.

2 Mestrando em Saúde Pública da Faculdade de Medicina da Universidade Federal de Minas Gerais (UFMG). Belo Horizonte. Minas Gerais. Brasil.

3 Professor Doutor do Curso de Medicina da Faculdade de Saúde e Ecologia Humana (FASEH). Vespasiano. Minas Gerais. Brasil.

4 Professor Titular da Universidade Federal de Minas Gerais (UFMG). Belo Horizonte. Minas Gerais. Brasil.

5 Professora Associada do Departamento de Pediatria da Faculdade de Medicina da UFMG. Belo Horizonte, Minas Gerais. Brasil. 
A febre é um sintoma de enfermidade comum na infância, sendo causa de procura de atendimento para consulta ao pediatra em serviços de emergência, ambulatórios ou consultórios particulares ${ }^{1-3}$.

19 a $30 \%$ das visitas ao pediatra nos serviços de urgência e emergência, se deve a essa sintomatologia ${ }^{1}$. Tal situação ocorre devido à associação de doença e febre, servindo como sinal alerta para aqueles que buscam o atendimento diante dessa circunstância. O sintoma febre é acompanhado da sensação de ansiedade, insegurança e preocupação por parte dos pais/ cuidadores $^{4}$. Concepções equivocadas sobre a febre, fazem com que procurem por esses serviços sem necessidade, pois consideram a febre como indicador de enfermidade, sendo assim prejudicial ${ }^{5-}$ 7. As preocupações sobre a febre costumam ser compostas por associações incorretas entre a febre e gravidade das doenças ${ }^{2}$.

Discrepância entre a literatura científica e concepções de febre necessitam ser exploradas assim como as definições atribuídas pelos pais acerca de febre elevada e perigosa, pois tais conceitos podem influenciar as suas práticas ${ }^{8}$.

A baixa compreensão da febre revela a necessidade de pesquisas que determinem com precisão o que os pais sabem sobre esse assunto, o grau de ansiedade e medo associados à febre infantil, para que futuramente se possa orientar com maior exatidão os pais sobre o manejo da febre ${ }^{9}$.

As ações dos pais/cuidadores são influenciadas através da história, e práticas diversas são aplicadas para o tratamento do paciente febril (indução de vômito, banho, uso de medicamentos, e outras). O medo irreal da febre ou "febre fobia" está relacionada à concepções surgidas no século $\mathrm{XIX}^{10}$. A busca para entender e orientar os pais no manejo da febre perdura até hoje e pesquisadores tentam entender os motivos dessa preocupação ${ }^{7}$. Etnia, cultura, crenças e fatores socioeconômicos podem influenciar as atitudes e práticas com respeito à saúde e doença, e consequentemente em relação ao manuseio da criança febril ${ }^{10}$.

Dados da literatura reforçam que a "febre fobia" permanece por todo o mundo $0^{7,8,10-12,14}$. Diante deste contexto torna-se necessário a realização de estudos que visem investigar o conhecimento dos pais em relação à febre infantil e métodos de manejo da febre, para que se possa identificar se os casos de procura aos serviços de emergência com queixa de febre, se enquadram dentro da perspectiva deste tipo de serviço, que é tratar o risco iminente de morte. Assim, será possível intervir de forma positiva e educativa no campo de saber desses indivíduos, para que saibam identificar o que é febre, quais os são os benefícios e danos, como cuidar de forma segura de uma criança febril em casa e quando se deve procurar um serviço de saúde diante dessa condição.

O presente estudo tem por objetivo verificar o conhecimento, crenças, fontes de informação, práticas e atitudes dos pais e cuidadores no manejo da febre infantil.

\section{MATERIAL E MÉTODOS}

Estudo descritivo transversal, de caráter quantitativo. Os participantes foram recrutados nos meses de setembro de 2010 a janeiro de 2011. O referencial teórico foi estabelecido por meio de artigos científicos sobre conhecimento, crenças, experiências, fontes de informação e prática dos pais no manejo da febre na infância. A busca de artigos foi realizada na Biblioteca Virtual em Saúde (BVS), nas bases de dados da Scientific Electronic Library Online (SCIELO), Literatura Internacional em Ciências da Saúde (MEDLINE) e Literatura LatinoAmericana e do Caribe em Ciências da Saúde (LILACS) e na National Library of Medicine (PubMed). Foram pesquisados e analisados artigos científicos dos anos de 1980 a 2016. Foram selecionados artigos publicados nos últimos 36 anos, para averiguar a importância e os avanços em relação ao manejo da febre infantil, desde a invenção do termo febre fobia por Schmitt em $1980^{11}$.

O estudo foi realizado no setor de acolhimento com classificação de risco, no prontoatendimento do Hospital Infantil João Paulo II pertencente à Fundação Hospitalar do Estado de Minas Gerais (FHEMIG), inseridos na rede pública de saúde. O serviço é aberto à comunidade, sendo a sua clientela procedente da região metropolitana de Belo Horizonte e dos municípios do interior do Estado de Minas Gerais.

A população do estudo foi composta por todos os pais e cuidadores que os pais e cuidadores procuraram o serviço de urgência com relato que a criança estava ou tinha apresentado febre nas últimas 72 horas. A amostra dessa pesquisa foi constituída por 286 pais/cuidadores. Para seleção da amostra, considerou-se o percentual de pais/ cuidadores que utilizavam o termômetro dentro da população do estudo. A unidade amostral foram os pais/cuidadores das crianças e o esquema amostral utilizado para coleta de dados foi a amostragem 
aleatória simples (AAS), sendo assim cada unidade amostral dentro da população tinha a mesma probabilidade de ser selecionado para fazer parte da amostra. Nesse estudo os pais/cuidadores, só poderiam ser selecionados uma única vez.

O cálculo amostral foi realizado pela fórmula de populações infinitas e amostragem aleatória simples:

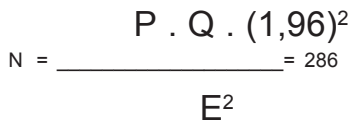

Para garantir o tamanho amostral, os cálculos foram feitos tendo como referência uma margem de erro de 5 pontos percentuais com $95 \%$ de confiança e a estimativa da proporção fixada em $76,7 \%$ das pessoas que utilizaram o termômetro.

Os participantes responderam um questionário contendo questões relacionadas ao conhecimento sobre o manejo da febre e características socioeconômicas dos entrevistados. O questionário construído teve como alicerce para estruturação das perguntas, o roteiro de uma entrevista semiestruturado utilizado em estudo na Austrália ${ }^{12}$. Posteriormente o instrumento foi adaptado a realidade dos pais brasileiros que constituíram a amostra dessa pesquisa.

O questionário possuía 33 questões. Sendo as questões de número 1 a 25 referentes ao manejo da febre na criança (métodos de aferição de temperatura, controle da febre, frequência de monitoramento da temperatura, procura por atendimento médico, crenças, conhecimentos, medicamentos utilizados, fontes de informação). E as questões de número 26 a 33 foram referentes a aspectos socioeconômicos (idade do entrevistado e da criança, número de filhos, estado civil, renda, escolaridade, tipo de residência, número de pessoas que habitam a residência, proximidade da residência a casa de parentes). As aplicações dos questionários ocorreram em turnos alternados manhã, tarde e noite, durantes os sete dias da semana.

Os dados foram tabulados e submetidos a análises uni- e bi-variadas com o auxílio do software SPSS (Statistical Package for the Social Science) 15.0 e STATA 10.0. A análise dos dados iniciou-se com a utilização de técnicas de análise descritiva, contendo a frequência absoluta e relativa e medidas resumo como média, mediana, desvio padrão, mínimo e máximo para a identificação das principais características pais/cuidadores e das crianças.
A avaliação da existência de associação entre as variáveis, que descrevem o conhecimento e a percepção dos pais/cuidadores sobre a febre com as variáveis socioeconômicas, foi realizada com o teste exato de Fisher. Tendo em vista, a existência de células nas tabelas, cujo valor esperado do teste Qui-Quadrado ficou abaixo de 5.

Para avaliar a existência de possíveis diferenças entre os grupos de entrevistados para variáveis contínuas como a idade das crianças, foi realizado o teste t-Student no caso de dois grupos. E utilizou-se a análise de variância (ANOVA) no caso de três ou mais grupos. Isso, em virtude da normalidade dos dados, verificada a partir do teste Shapiro Wilk e Kolmogorov Smirnov, além do gráfico de probabilidade normal e do histograma, que não indicaram desvios significativos dos dados em relação a distribuição normal.

Para determinar se as diferenças e associações encontradas foram estatisticamente significativas, utilizou-se o nível de significância de $5 \%$. Assim, consideraram-se como significativas diferenças e associações, cuja probabilidade de significância do teste $p$-valor, foi menor ou igual a 0,05 .

Os critérios de inclusão foram: idade superior a 18 anos, autorização do participante constando a assinatura do mesmo, nacionalidade brasileira, capacidade de ler e conversar em português, pais de crianças de 0 a 12 anos referente à faixa etária de atendimento do Hospital Infantil João Paulo II, preenchimento acima de $20 \%$ das respostas do questionário. Os critérios de exclusão foram: pais/cuidadores que questionários obtiverem índices de preenchimento resposta inferiores a 20\% e que os filhos apresentavam idade igual ou superior a 13 anos. Não houve recusa de pais/cuidadores em participar do estudo.

O recrutamento dos pais e cuidadores aconteceu anteriormente à consulta médica, no momento em que seus filhos estavam febris ou que acreditavam que a criança estava com febre. Tal conduta foi tomada para que fossem ouvidos quando estavam com medo e apresentavam preocupação com a febre, com intuito de observar as consequências acarretadas por esses sentimentos, que poderiam influenciar as condutas e atitudes. $A$ fala dos pais colhida no momento em que seus filhos estão com febre tem maior importância clínica, devido ao fator de ansiedade e apreensão frente a febre de seus filhos. Também a incompreensão do desenvolvimento do sintoma leva à exacerbação do medo e a concepções equivocadas ${ }^{12,13}$. 
Previamente a aplicação do questionário, os pais ou cuidadores eram informados quanto aos objetivos da pesquisa por meio da apresentação e assinatura do termo de consentimento livre e esclarecido (TCLE). O estudo foi aprovado pelo Comitê de Ética em Pesquisa da Universidade Federal de Minas Gerais, com o parecer número ETIC 0037.0.287.203-10 e no Comitê de Ética em Pesquisa da Fundação Hospitalar de Minas Gerais com o parecer número 038/2010.

\section{RESULTADOS E DISCUSSÃO}

Os pais e cuidadores selecionados para fazer parte desse estudo representaram amplo espectro socioeconômico. $48,6 \%$ era casado. $52,8 \%$ residia com o cônjuge, e em suas casas moravam de três a quatro pessoas. $71,3 \%$ possuíam casa própria e $70,3 \%$ residiam próximo aos pais ou parentes. A proximidade com a casa de familiares pode ser um fator que influencie as condutas e práticas frente à febre na criança, devido a possibilidade de compartilhar experiências e práticas.

$45,6 \%$ possuíam o ensino médio, $43 \%$ ensino fundamental completo e apenas $2,4 \%$ curso superior. Em pesquisa realizada no Brasil, no município de São Paulo, nenhum dos participantes tinham cursado faculdade e a maior parte deles possuía o ensino fundamental incompleto $(40 \%)^{9}$. Estudo realizado na Itália, observou-se que a preocupação exagerada das mães diante da febre estava associada ao baixo nível educacional14. Entretanto nessa pesquisa verificamos que o nível de escolaridade não é baixo, o que pode sugerir a presença de outros fatores que podem interferir nas concepções em relação à febre infantil.

$54 \%$ tinham renda mensal familiar de até dois salários mínimos, $25,5 \%$ de 2,1 a 3 salários, $8,5 \%$ não possuía renda mensal fixa, $7,3 \%$ renda de 3,1 a 5 salários e apenas $1,7 \%$ recebiam mais de 5,1 salários mínimos por mês. Cabe ressaltar que o estudo foi desenvolvido em hospital público que atende pacientes do Sistema Único de Saúde (SUS), o que poderia explicar o fato de grande parcela dos entrevistados possuírem renda mensal de até dois salários mínimos e apenas sete pais/ cuidadores $(2,4 \%)$ possuírem ensino superior.

$42 \%$ possuíam um filho, $32 \%$ tinham dois e $15 \%$ três filhos, sendo que aproximadamente $90 \%$ até três filhos. Dados semelhantes aos encontrados em outro estudo, sugerindo assim, pouca experiência com suas crianças? ${ }^{9}$.
$98,6 \%$ das crianças conduzidas ao serviço de urgência se encontravam na faixa etária de um a seis anos de idade. $89,9 \%$ das crianças encaminhadas para atendimento médico foram levadas pelas mães. A preocupação faz com que as mães mudem suas rotinas diárias, rumo a atendimento médico para seus filhos ${ }^{15}$.

Quanto ao tempo que aguardavam para procurar por atendimento médico, 23,3\% aguardavam de 24 a 36 horas, $22,1 \%$ de 12 a 24 horas, $14,5 \%$ procuram imediatamente e apenas $12,1 \%$ aguardam de 48 a 72 horas. A idade da criança e o número de filhos, não foram estatisticamente significativos relacionado ao tempo em que procuram por atendimento. A má compreensão do manejo da criança febril foi reforçada em estudo, o qual $58,3 \%$ mães relataram que levavam seus filhos ao médico após algumas horas do aparecimento da febre ${ }^{9}$. A investigação imediata da febre é necessária, quando apresenta duração maior que três dias (acima de 72 horas). As infecções virais têm "direito" a três dias de febre. Ultrapassados os três dias, pode-se pensar em infecção bacteriana ${ }^{4}$.

$70,6 \%$ pais/cuidadores possuíam termômetro e $29,4 \%$ não possuíam o instrumento e detectavam a febre pela palpação das partes do corpo. Outra parcela dos entrevistados identificava a febre através de sinais como irritação $(1,2 \%)$, vermelhidão $(1,2 \%)$ ou quando a criança estava quente e quieta $(1,2 \%)$. Estudo realizado em São Paulo, com 60 mães, 58 tocavam a pele em locais como cabeça e pescoço e 46 utilizavam o termômetro para detectar a febre ${ }^{9}$. Pesquisa realizada no estado de Santa Catarina, apresentou dados próximos em que $25,8 \%$ utilizavam o método palpatório, $74,2 \%$ o termômetro e $92,7 \%$ tinham o termômetro em casa ${ }^{16}$. Em contrapartida, outro estudo feito no ano de 1985,38 a $44 \%$ dos pais não tinham o termômetro em casa ${ }^{17}$. Evidenciando o aumento de acesso ao termômetro.

$84,6 \%$ possuía o termômetro digital se comparado ao de mercúrio (13,9\%). A preferência se deu pela maior facilidade no manuseio e leitura. $76,6 \%$ sabiam manusear o termômetro. Quanto ao intervalo de tempo gasto para a retirada do termômetro de mercúrio, apenas $51,6 \%$ procederam com as normas preconizadas deixando o termômetro na criança de 3 a 5 minutos ${ }^{18,19}$. $29 \%$ deixavam acima de 5 minutos e $19,4 \%$ de 1 a 3 minutos. 95,3\%, retiravam o termômetro digital após o sinal sonoro. Aproximadamente a metade dos entrevistados $(48,4 \%)$, não observaram o tempo 
adequado para a retirada do termômetro de mercúrio. Mostrando que a medida de temperatura nem sempre é confiável, conforme outros estudos que afirmam que o fato de possuir termômetro em casa não prevê a habilidade de leitura ${ }^{17,7,20}$. Os termômetros digitais devem permanecer até a ocorrência de sinal audível, caso contrário o valor concedido pode não ser confiável ${ }^{18,19}$.

A escolaridade e status socioeconômico não interferiram em saber manusear o termômetro. Diferentemente de outros dois estudos, em que baixo status socioeconômico foi associado a inabilidade de leitura do termômetro e maior escolaridade com maior acurácia na leitura do termômetro de mercúrio ${ }^{17,20}$. Quanto a via de aferição de temperatura preferencial foi a axilar $(81,5 \%)$, apesar da literatura relatar que a medida mais confiável é aquela aferida pelas vias oral ou retal ${ }^{1}$. Diferentemente do estudo realizado na Dinamarca no ano de 2016, com uma amostra de 15 pais, em que 13 utilizavam o termômetro via retal21.

Quanto ao valor considerado como febre, $34,3 \%$ responderam que corresponde à febre temperaturas de $37,5^{\circ} \mathrm{C}$. Temperaturas axilares consideradas fisiológicas estão entre 35,5 e $37,5^{\circ} \mathrm{C}^{19}$. A literatura traz valores considerados como febre pelos pais e revela diferenças culturais quanto a conceitos incorretos sobre o diagnóstico de febre, semelhantes aos dados obtidos nessa pesquisa. Em estudo realizado na Austrália, 51\% consideravam febre valores entre $38,0^{\circ} \mathrm{e} 38,5^{\circ} \mathrm{C}$, na Noruega, $21 \%$ temperaturas entre $37,0^{\circ}$ e $38,5^{\circ} \mathrm{C}$, na Itália acima de $37,0^{\circ} \mathrm{C}$, na Arábia Saudita $30 \%$ consideram $38,0^{\circ} \mathrm{C}$ e em outro estudo no Brasil $40 \%$ conceituavam febre como temperaturas de $38,0^{\circ} \mathrm{C}$ $8,22,14,13,9$

Para controle da febre $96,3 \%$ administravam antitérmicos, com maior frequência a dipirona $(50,5 \%)$ e o paracetamol (39,3\%). O ibuprofeno foi usado por $1,1 \%$ dos pais e o uso do ácido acetilsalicílico (AAS) foi relato por $0,4 \%$ dos pais. Semelhantes aos dados de outro estudo, em que $100 \%$ recebiam antitérmicos e a dipirona era usada em $73,3 \%$ dos casos e o paracetamol em $45 \%$. Na Itália foi identificado que o paracetamol foi o mais utilizado pelas mães $(81,2 \%), 1,1 \%$ usava dipirona e $1,1 \%$ o AAS ${ }^{14}$. No Canadá a medicação de escolha é o paracetamol, $85,6 \%$ dos pais o utilizam em episódio de febre ${ }^{23,24}$. Nos países europeus e a América do Norte a dipirona não é usada sob a alegação de produzir agranulocitose ${ }^{25}$.

Dos pais que afirmaram que administravam antitérmicos, $83,3 \%$ relataram desconhecer os efeitos adversos dessas medicações, diferentemente do estudo realizado em São Paulo, em que $71,6 \%$ relataram que esses medicamentos poderiam causar efeitos como alergia, vômitos e náuseas ${ }^{9}$.

Somente temperaturas entre $38,5^{\circ}, 39,0^{\circ} \mathrm{e}$ $40,0^{\circ} \mathrm{C}$ ou maiores, deveriam ser reduzidas com medicamentos, a administração de antipiréticos deve ser individualizada e baseada em outros sinais além da temperatura ${ }^{9}$. Existem evidências que a febre estimula as defesas do organismo, não existe risco pela febre alta em si, a não ser em crianças muito debilitadas, cardiopatas ou com insuficiência respiratória. Por outro lado, lesões do sistema nervoso podem ocorrer em níveis próximos de $42,5^{\circ} \mathrm{C}$ e em crianças suscetíveis pode causar convulsões ${ }^{4}$.

Neste estudo, também foi avaliado se o número de filhos, escolaridade e renda mensal interferiam na administração dos antitérmicos. A quantidade de filhos e a renda mensal familiar não foram significativos quanto a administração desse tipo de medicação. Entretanto a associação com escolaridade apresentou-se significativa, pais com maior escolaridade, que possuem até o ensino médio administram mais antitérmicos $(46,9 \%)$. Esse fato sugere que a experiência educacional pode ter influenciado significativamente o conhecimento dos entrevistados ${ }^{8}$.

Quanto à dosagem de antitérmicos administrados na criança febril, $90 \%$ dos entrevistados revelaram usar como parâmetro o peso, $3,6 \%$ consideravam a idade da criança, $3,3 \%$ receitas médicas. A maioria dos pais e cuidadores usavam como parâmetro $1 \mathrm{gota} / \mathrm{Kg} /$ dose tanto para dipirona como para paracetamol. A adequação da dose de cada antitérmico estabelecida pela literatura como faixa terapêutica, é de 10 e $15 \mathrm{mg} /$ $\mathrm{kg}$ para o paracetamol, 10 a $20 \mathrm{mg} / \mathrm{kg} /$ dose para a dipirona ${ }^{26}$. O conhecimento dos pais sobre a eficácia e dosagem apropriada dos anti-piréticos é questionável ${ }^{7}$.

$14 \%$ pais/cuidadores também administravam antibióticos nas crianças com febre. Em outro estudo no Brasil, 3,3\% administravam antibiótico em crianças febris sem conhecimento exato da causa e prescrição médica ${ }^{9}$. O antibiótico de escolha foi a amoxicilina (70\%). Foi possível perceber o uso indiscriminado dessas medicações pela população estudada, entretanto quando questionados sobre os efeitos colaterais dessas medicações, $65,7 \%$ relataram não conhecê-las. Esses dados demonstram o pouco conhecimento 
dos pais em relação a esse assunto ${ }^{9}$. Dos entrevistados que não administravam antibióticos, $43 \%$ tinham apenas um filho, sugerindo que a pouca experiência pode ter levado a uma menor administração dessa medicação. A coleta de dados deste trabalho foi iniciada em setembro/2010. É possível que este tipo de medicamento não tenha sido utilizado em maior escala, em razão da Resolução da ANVISA no 20 de 05/05/11 que regulamentou o controle de medicamentos à base de substâncias classificadas como antimicrobianos, exigindo para a venda a apresentação de receita médica ${ }^{27}$.

Dos métodos não farmacológicos, apenas $5,2 \%$ dos entrevistados não os utilizam para controle da febre. $73,8 \%$ davam banho, $9,4 \%$ banho e aplicação de compressa embebida em álcool, 4,2\% aplicações de compressas embebidas em álcool e $2,4 \%$ aplicação de compressa de água fria. 96,6\% associavam métodos não farmacológicos com farmacológicos. A literatura internacional aponta que os pais controlam ou reduzem a febre com métodos não farmacológicos, farmacológicos ou ambos. Os não farmacológicos incluem remoção de roupas, ingestão de líquidos, aplicação compressão frias ou mornas e banho ${ }^{12}$.

Banho, compressas frias e aplicação de álcool na pele não tem valor quando usados isoladamente, pois não atuam no mecanismo fisiopatológico da febre. Mas uma vez que o ponto da termorregulação hipotalâmico tenha sido rebaixado farmacologicamente e, se for ainda necessário, depois da retirada de agasalhos e permanência em ambiente ventilado, pode-se recorrer a banhos mornos de imersão por 10 a 20 minutos ou friç̧ão delicada com esponja umedecida em água morna em partes do corpo por até 20 a 30 minutos. Esse processo somente deverá ser utilizado se trouxer conforto para a criança e não causar mais problemas para os pais. A água fria pode causar calafrios e tremores, que além do desconforto, aumentam a temperatura. O álcool pode ser absorvido pela pele e causar toxicidade sistêmica e nunca deve ser utilizado ${ }^{4}$.

$43 \%$ dos entrevistados, consideravam a febre como sinal de alerta, mostrando que há algo de errado com o organismo e $37 \%$ como sinal de infecção. A febre tem a utilidade de servir como sinal de alerta, e estima-se que $20 \%$ a $30 \%$ das consultas pediátricas têm a febre como queixa única preponderante ${ }^{4}$. A febre é considerada benéfica, pois é um aviso, um sinal de alerta para os pais que está acontecendo alguma coisa de errado no corpo da criança $^{12}$. É uma resposta adaptativa do organismo em resposta a invasão de microrganismos ou outros pirógenos conhecidos. A febre leve a moderada está associada com resposta imunológica mais intensa e desempenha papel fundamental no processo de resposta antiinflamatória ${ }^{8}$. Evidenciando nesta pesquisa o conhecimento da febre relacionado às defesas orgânicas. Estudo realizado em São Paulo, mostrou que $91,7 \%$ pais consideravam a infecção como a principal causa da febre. É um consenso entre os pediatras que mais de $90 \%$ das febres agudas em crianças são oriundas de infecções virais ${ }^{28}$.

$70 \%$ dos pais consideravam que a febre trazia malefícios como: sinal de infecção, sinal de algo ruim, sinal de gravidade, perda de apetite, sinal de doença, convulsão, prostração, alteração do comportamento, dados semelhantes ao estudo realizado no Brasil. ${ }^{9}$ 97,6\% consideravam a febre perigosa, pois acreditavam acarretar complicações como hemorragias, parada cardiorrespiratória, pneumonia, etc. Possibilitando, assim, compreender os motivos pelos quais os pais apresentam medo da febre.

Quanto ao principal dano em decorrência da febre, $70 \%$ citaram a convulsão. Complicações como sonolência $(17,8 \%)$, prostração $(15,7 \%)$, desidratação $(7 \%)$ e danos cerebrais $(3,8 \%)$ também foram expostos. $0,7 \%$ relataram que a febre não acarreta danos. Esses dados estão em conformidade com outros estudos ${ }^{-9,13}$.

Para manejo da febre fontes de informação eram utilizadas, dentre essas $17 \%$ recorriam a internet, $16,4 \%$ televisão, $8,4 \%$ livros, 5,9\% em jornais e $0,3 \%$ leitura da bula dos medicamentos, $0,3 \%$ rádio. $37 \%$ não utilizavam veículos de informação. $54 \%$ buscavam informações com profissionais de saúde, $30 \%$ familiares, $8 \%$ amigos/ vizinhos e $5 \%$ com pessoas mais experientes. Alguns ainda utilizavam como fontes de informação para decidir medicações para controle da febre, o farmacêutico $(36,7 \%)$ e o balconista da farmácia $(5,7 \%)$. Em conformidade com estudo realizado na Dinamarca, em que os pais também usavam como fontes de informação, os profissionais de saúde, livros, família, amigos, internet, mas também se baseiam no senso comum, na experiência e intuição ${ }^{21}$.

Os pais aprendem o manejo da febre por meio de diferentes fontes, mas observou-se a preferência por profissionais de saúde, devido considerarem que as informações dadas por esses profissionais são mais verdadeiras e que em alguns 
sites da internet a acurácia das informações são questionáveis $^{12}$. Nesta pesquisa os pais utilizaram como fontes de informação tanto pessoas como veículos, independentemente de sua renda mensal familiar e escolaridade. As fontes de informação têm forte influência no conhecimento e práticas dos pais em relação à febre, o conhecimento sobre os benefícios, embora limitados são obtidos com profissionais de saúde. A interminável busca por informações por meio de uma variedade de fontes pode criar problemas adicionais. Informações de familiares, amigos, livros e profissionais de saúde frequentemente são contraditórias. Os conflitos de informação aumentam as preocupações acerca da febre, e podem promover sentimentos de insegurança ${ }^{7}$.

\section{CONCLUSÃO}

Esse estudo permitiu verificar que pais/ cuidadores possuem percepções errôneas frente à febre. O medo exacerbado gera preocupações que associadas as suas concepções direcionam condutas e práticas de manejo da febre. Assim como identificado em outros países a febre fobia persiste, permeada por sentimentos de insegurança para cuidar de uma criança nessa condição. Ficou evidente que conhecimentos e saberes são elaborados e compartilhados socialmente em relação à febre infantil. Esses conhecimentos transitam pela comunicação, fontes de informação utilizadas e experiência adquirida ao longo de suas vidas, direcionando condutas, comportamentos, sentimentos e crenças. A influência cultural foi fator marcante na definição de conceitos e crenças sobre a febre. A convulsão foi o principal dano apontado na presença de febre. Para prevenir danos, pais/ cuidadores utilizam métodos farmacológicos e não farmacológicos. Dessa forma dão início ao tratamento com o uso de medicações, principalmente antitérmicos. Também recorrem ao banho, ingestão de líquidos e recursos populares. A febre é indicativo de doença e muitos a consideram sinal de infecção. Cuidar de uma criança febril é uma fase emocionalmente desafiadora para os pais. É importante desmitificar algumas crenças negativas e concepções errôneas para que os pais e cuidadores tenham tranquilidade e saibam lidar com segurança de uma criança febril, e compreendam quando é necessário buscar por atendimento médico.

\section{REFERÊNCIAS}

1. Trotta EA, Gilio AE. Febre aguda sem sinais de localização em crianças menores de 36 meses de idade. J Pediatr (Rio J). 1999; 75(2): 214-22.

2. Consindine J, Brennan D. Effect of an evidence-based paediatric fever education program on emergency nurses' knowlegde. Accid Emerg Nurs. 2007; 15(1):1019.

3. Alves JGB, Almeida NDM de, Almeida CDCM de. Banho tépido e dipirona versus dipirona isolada no tratamento de crianças com febre. São Paulo Med. J. 2008; 126(2):107-11.

4. Murahovschi J. A Criança com febre no consultório. J Pediatr (Rio Janeiro). 2003; 79(1):55-64.

5. Krantz C. Childhood fevers: developing an evidencebased anticipatory guidance tool for parents. Pediatri Nurs. 2001; 27(6):567-71.

6. Matziou V, Brokalaki $H$, Kyritsi $H$, Perdikaris $P$, Gymnopoulou E, Merkouris A. What Greek mothers know about evaluation and treatment of fever in children: An interview study. Int J Nurs Stud. 2008; 45(6):829-36.

7. Walsh A, Edwards $\mathrm{H}$. Management of childhood fever by parents: literature review. J Adv Nurs. 2006; 54(2):217-27.

8. Walsh A, Edwards H, Fraser J. Parent's childhood fever management: community survey and instrument development. J Adv Nurs. 2008; 63(4): 376-78.

9. Rocha CT, Regis RR, Nelson-Filho P, Queiroz AM de. Febre na infância: conhecimento, percepção e atitude materna. Rev Odontol Univ São Paulo. 2009; 21(3):24451.

10. Tessler H, Gorodischer R, Press J, Bilenko N. Unrealistic concerns about fever in children: the influence of cultural-ethnic and sociodemografic factors. Isr Med Assoc J. 2008; 10(5):346-49.

11. Schmitt BD. Fever phobia: misconceptions of parents about fever. Am J Dis Child. 1980; 134(2): 176-81.

12. Walsh A, Edwards H, Fraser J. Influences on parents' fever management: beliefs, experiences and information sources. J Clin Nurs. 2007; 16(12):2331-40.

13. Al-eissa YA, Al-Sanie AM, Al-Alola SA, Al-Shaalan MA, Ghazal SS, Al-Harbi AH, et al. Parental perceptions of fever in children. Ann Saudi Med. 2000, 20(3-4): 202-5.

14. Impicciatore PMD, Nannini SPD, Pandolfini CBA, Bonati MMD. Mothers' Knowledge of, Attitudes toward, and Management of Fever in Preschool Children in Italy. Preventive Medicine. 1998; 27(2): 268-73.

15. Blumenthal I. What parents think of fever. Fam Pract; 1998; 15(6):513-8.

16. Feldahaus T, Cancelier ACL. Conhecimentos dos pais sobre febre em crianças. Arq. Catarin. Med. 2012; 41(1):16-21.

17. Fischer $\mathrm{H}$, Moore K, Roaman RR. Can mothers of infants read a thermometer? Clin Pediatr (Phila). 1985; 24:120.

18. Koch RM, Horiuchi LMO, Paloschi IM, Ribas MLV, Motta HS, Walter RL. Técnicas básicas de enfermagem. 20ª.ed. Curitiba: Século XXI; 2004

19. Potter P. Semiologia em enfermagem. $4^{a}$.ed. Rio de Janeiro: Reichmann \& Affonso; 2002. 
20. Porter RS, Wenger FG. Diagnosis and treatment of pediatric fever by caretakers. J Emerg Med. 2000; 19(1): $1-4$

21. Kelly M, Sahm LJ, Shiely F, O'Sullivan R, McGillicuddy A, McCarthy S. Parenteral knowledge, attitudes and beliefs regarding fever in children: a interview study . BMC Public Health. 2016; 16:540.

22. Eskerud JR, Hoftvedt BO, Laerum E. Fever: knowledge, perception and attitudes. Results from a Norwegian population study. Fam Pract, 1991; 8(1):32-6.

23. Karwowska A, Nijssen-Jordan C, Johnson D, Davies HD. Parental and health care provider understanding of childhood fever: a Canadian perspective. CJEM, 2002; 4(6):394-400

24. Erkek N, Senel S, Sahin M, Ozgur O, Karacan C. Parents perspectives to childhood fever: Comparison of culturally diverse populations..J Paediatr Child Health. 2010; 46(10): 583-7.

25. Danieli $P$, Leal MB. Avaliação da segurança da dipirona: uma revisão. Rev Bras Farm. 2003, 84(1):17-20.
26. Sukiennik R, Halpern R, Manica JLL, Plentz FD, Bergamin G, Loss L, Ayres MV, Dalphiane KP. Antitérmicos na emergência pediátrica: estamos usando a dosagem adequada? Pediatria (são Paulo). 2006; 28(3):175-83.

27. Ministério da Saúde, Agência Nacional de Vigilância Sanitária, Resolução-RDC № 20, de 05 de maio de 2011 [Internet]. Brasília, Brasil: Ministério da Saúde, 2011. [citado em 22 nov. de 2016].

28. Casey R, McMahon MC, Pasquariello PS Jr, Zavod W, King FH Jr. Fever therapy: an educational intervention for parents. Pediatrics. 1984; 73(5): 600-5.

\section{Correspondência}

Ana Carolina Micheletti Gomide Nogueira de Sá

Rua Castigliano, $n^{\circ} 283$, apartamento 202, Bairro Padre Eustáquio. Belo Horizonte.

CEP 30720-402.

Belo Horizonte - Minas Gerais - Brasil.

Email: carolmichelettigomide@gmail.com 